\title{
Preface
}

\author{
Chunxiang Xu
}

Published online: 13 March 2014

(c) Science China Press and Springer-Verlag Berlin Heidelberg 2014

Wide bandgap semiconductors play more and more important roles in both scientific researches and industrial applications. The unique wide bandgap properties provide a platform for flexible functional design for crystal growth, defect engineering, interface engineering, and device construction. Meanwhile, the underlying physical mechanism of lattice and energy band match, interface strain, and charier charge injection and transport inspire the theoretical and experimental investigation for deep understanding. The broad applications also attract considerable industrial and commercial interesting in green lighting, communication, environmental monitoring, spatial technology, biomedicine, and so on. In fact, the research and development passion on the wide bandgap semiconductors has been radiating and spreading in worldwide. In this area, Chinese scientists have paid great effort and made outstanding achievement in material growth and epitaxy technology, electrical and optoelectronic characterization and optimization, and functional design and device development.

In this situation, we organize a special issue focused on the topic of wide bandgap semiconductor materials and optoelectronic devices to review the research progress supported by the journal of Chinese Science Bulletin. Twelve representative research groups of China are invited, and eight groups completed their contribution finally through the regular international peer view, which include five review papers and three original research reports.

SPECIAL TOPIC: Wide Bandgap Semiconductor Materials and Devices

\section{C. $\mathrm{Xu}(\bowtie)$}

State Key Laboratory of Bioelectronics, School of Biological

Science and Medical Engineering, Southeast University,

Nanjing 210096, China

e-mail: xcxseu@seu.edu.cn
Besides briefly reviewing the international progress in this area, the review papers summarize systematically the serials research works done by them and exhibit their novel contribution. For examples, Huang et al. [1] developed a skillful crystal growth technique and obtained high-quality single crystal $\mathrm{ZnO}$. Facing to the bottleneck of p-type $\mathrm{ZnO}$, they further explored a conceptual approach for p-type doping. As an alternative strategy to overcome the p-type problem, Liu et al. [2] reviewed the progress in $\mathrm{ZnO}$-based heterojunction ultraviolet light-emitting diodes (LEDs) and laser diodes (LDs) involving thin films and nanostructures. As important and representative wide bandgap semiconductors, III-nitrides have been widely and deeply investigated [3-7]. In this special issue, some innovations of the growth and processing technologies are laid out; for instances, pulsed metal organic chemical vapor deposition has been developed by Hao et al. [3], and hydride vapor phase epitaxy, laser lift-off, and chemical mechanical polishing techniques have been modified in Zhang et al. [4]. Based on their improved techniques, the high-quality materials, such as GaN, InN, InAlN, and AlGaN were obtained, and the corresponding advanced devices, such as high electron mobility transistor, vertical structure LEDs, and LDs, were successfully fabricated. Zhang et al. [5] present a very attractive subject, GaN-based optoelectronic devices on Si substrate, which would act as a platform for mature Si-based integrated circuits and even for optoelectrical integration. Besides the typical III-V GaN-based and II-VI ZnO-based compounds, some other wide bandgap semiconductors are also investigated in this special issue. For examples, Zhang et al. [6] employed ion beam assisted deposition and ion implantation to fabricate the sulfur-doped cubic boron nitride thin film and revealed optimized electrical properties. Jun Xu's groups [7, 8] displayed $\mathrm{SnO}_{2}: \mathrm{Eu}^{3+}$ photoluminescence in an effective 
Förster resonant energy transfer. The physical process and mechanism beyond the nice crystallization and advanced device performance are also explored in these papers.

I would appreciate all authors for their contribution for this special topic. It is expected to provide a valuable reference to the colleagues to catch the evolution, understand the mechanism, and outlook the future trend.

\section{References}

1. Huang F, Lin Z, Lin WW et al (2014) Research progress in ZnO single crystal: growth, scientific understanding and device applications. Chin Sci Bull 59:1235-1250

2. Liu YC, Xu HY, Liu CY et al (2014) Recent progress in ZnObased heterojunction ultraviolet light-emitting devices. Chin Sci Bull 59:1219-1227
3. Hao Y, Xue JS, Zhang JC (2014) Pulsed metal organic chemical vapor deposition of InAlN-based heterostructures and its application in electronic devices. Chin Sci Bull 59:1228-1234

4. Zhang GY, Shen B, Chen ZZ et al (2014) GaN based substrates and optoelectronic materials and devices. Chin Sci Bull 59:1201-1218

5. Zhang BJ, Liu Y (2014) A review of GaN based optoelectronic devices on silicon substrate. Chin Sci Bull 59:1251-1275

6. Zhang XW, Yin ZG, Si FT et al (2014) Electrical properties of sulfur-implanted cubic boron nitride thin films. Chin Sci Bull 59:1280-1284

7. Jiang R, Yan DW, Lu H et al (2014) Reverse leakage current in AlGaN-based ultraviolet light-emitting diodes. Chin Sci Bull 59:1276-1279

8. Zhang XW, Zhang P, Lin SB et al (2014) Energy transfer process between $\mathrm{Eu}^{3+}$ and wide-band-gap $\mathrm{SnO}_{2}$ nanocrystals in silica films studied by photoluminescence excitation and time-resolved photoluminescence techniques. Chin Sci Bull 59:1285-1290 